\title{
Neonatal thrombocytopenia, maternal SLE related or flu like syndrome?
}

\author{
Eirini Koutsounaki*, Kaliopi Drakaki, Xenia Bitouni, Anastasia Kapetanaki, Margarita Tzaki and Heraklis Salvanos \\ Helena Venizelou General Hospital of Athens, NICU, Greece
}

A baby girl born to a mother with SLE, receiving prednisolone and hydroxychloroquine treatment through pregnancy, developed severe thrombocytopenia after the fourth day of life. The late preterm neonate GA: $35^{+4}$ weeks, BW $2510 \mathrm{~g}\left(50^{\text {th }}\right)$ AGA was born by uncomplicated cesarean section due to previous cesarean and birth initiation. The laboratory tests examined on the first day of life, due to transient tachypnea, showed a hemoglobin level of $17,5 \mathrm{~g}$ per deciliter, a platelet count of 237.000 per cubic millimeter and a white-cell count of 13.910 per cubic millimeter with 63 per cent neutrophils. On the fifth day of age, after an episode of fever $38^{\circ} \mathrm{C}\left(100.4^{\circ} \mathrm{F}\right)$ and lethargy, peripheral blood and cerebrospinal fluid was obtained for cultures and type. The C-reactive protein was only moderately elevated to $16 \mathrm{mg}$ per liter for one day. A complete blood count showed an increase in the white-cell count of 21.240 per cubic millimeter with 43 per cent neutrophils. The level of hemoglobin remained at $17.1 \mathrm{~g}$ per deciliter while the platelet count was 9.000 per cubic millimeter and further diminished to a nadir of 5.000 per cubic millimeter on the following day, despite the prompt initiation of treatment with intravenous immunoglobulin and random donor platelet transfusion. The baby was only moderately active with affected general appearance, peripheral petechial hemorrhages and bleeding at sites of venipuncture, but not from the mucosal surfaces. The central nervous system ultrasound neither revealed focal findings nor intraventricular hemorrhage. Wide spectrum antibiotics had initiated with the origin of the clinical signs, in case of sepsis (not proven). The condition complicated with DIC, with low fibrinogen and d-dimmer increase. The complete treatment included 2 grams of intravenous immunoglobulin (divided into 4 doses), transfusions of random donor platelets (two times), fresh frozen plasma and red blood cells. Normal findings of bone marrow aspiration, performed on the tenth day of age, was prerequisite, according to the hematologist's opinion, in case of the need of steroids additionally to treatment. The neonate condition gradually improved with a platelet count of 100.000 per cubic millimeter on the fifteenth day of life, no steroids administered. The child's mother, 34-year-old gravida II, no misconceptions referred, had received a diagnosis of systemic erythematosus lupus a few months prior to the present pregnancy conception. Her serologic testing was positive for ANA, anti-DNA, RNP, SSA (Ro) and negative for SSB (La), low C3, and low $\mathrm{C} 4$ in accordance with the primary diagnosis. She was treated with low dose of systemic corticosteroids and hydroxychloroquine and had no exacerbation of the disease during pregnancy, unaffected levels of her own platelets, besides no signs of intrauterine growth restriction

Copyright: (C2017 Boubacar S. This is an open-access article distributed under the terms of the Creative Commons Attribution License, which permits unrestricted use, distribution, and reproduction in any medium, provided the original author and source are credited. referred. Maternal antibodies crossing the placenta can lead to the clinical manifestations of neonatal lupus. The child's serology showed positive SSA (Ro) and negative for SSB (La) confirming the diagnosis of neonatal lupus. Serial electrocardiograms during the first weeks of age showed normal sinus rhythm.

\section{Discussion}

With the description of this case we aim to give an insight of the possible pathogenesis of the sepsis like syndrome our patient developed after the first days of life. The condition primarily originates from the mother's SLE and as the presence of the specific autoantibodies may impose. Any future children of these parents are at risk for neonatal lupus and congenital heart block.

The girl's mother pharmaceutical treatment might have contributed to the maintenance of the maternal as well as the fetal platelets within normal levels before birth, but according to pharmacokinetics this medication should have gradually 'washed out' during the next days after birth. On the other hand, the intrinsic catecholamine levels, related to the delivery and the short period of transient tachypnea, in this case, normalize soon that might have played a role by exposing the platelets to the maternal antibodies. In this context, the immunological cascade might have started leading to low neonatal platelet levels, peripheral bleeding, fibrinogen consumption and DIC initiation. Although an additional environmental factor, such as microbial infection, could not be proven from the several cultures and the mild increase of C- reactive protein, this could also not be excluded in terms of a viral infection since the father, who developed flu like symptoms, was visiting the newborn under strict hygiene measures in due course. In the long term follow up, at one and three months of age, no further complications were referred.

\section{References}

1. McCarthy PM, Bhudia SK, Rajeswaran J, Hoercher KJ, Lytle BW, et al. (2004) Tricuspid valve repair: durability and risk factors for failure. J Thorac Cardiovasc Surg 127: 674-685. [Crossref]

2. Ohata T, Kigawa I, Yamashita Y, Wanibuchi Y (2000) Surgical strategy for severe tricuspid valve regurgitation complicated by advanced mitral valve disease: long-term outcome of tricuspid valve supra-annular Implantation in eighty-eight cases. $J$ Thorac Cardiovasc Surg 120: 280-283. [Crossref]

3. Baraki H, Saito S, Al Ahmad A, Fleischer B, Haverich A, et al. (2015) Beating heart versus arrested heart isolated tricuspid valve surgery. Int Heart $J$ 56: 400-407. [Crossref]

Correspondence to: Eirini Koutsounaki, Helena Venizelou General Hospital of Athens, NICU, Greece, Tel: 2106402133, E-mail: eirkou@hotmail.com

Received: June 29, 2017; Accepted: July 21, 2017; Published: July 25, 2017 